Article

\title{
In Situ Monitoring of Growth of Vertically Stacked h-BN/Graphene Heterostructures on Ni Substrates and Their Interface Interaction
}

\author{
Wei Wei ${ }^{1,2}$, Guanhua Zhang ${ }^{3}$, Jiaqi Pan ${ }^{1}$, Yi Cui ${ }^{1, *(\mathbb{D})}$ and Qiang Fu ${ }^{2, * \mathbb{C}}$ \\ 1 Vacuum Interconnected Nanotech Workstation, Suzhou Institute of Nano-Tech and Nano-Bionics, \\ the Chinese Academy of Sciences, Suzhou 215123, China; wwei2018@sinano.ac.cn (W.W.); \\ jqpan2018@sinano.ac.cn (J.P.) \\ 2 State Key Laboratory of Catalysis, iChEM, Dalian Institute of Chemical Physics, \\ the Chinese Academy of Sciences, Dalian 116023, China \\ 3 State Key Laboratory of Molecular Reaction Dynamics, Dalian Institute of Chemical Physics, \\ the Chinese Academy of Sciences, Dalian 116023, China; zhanggh@dicp.ac.cn \\ * Correspondence: ycui2015@sinano.ac.cn (Y.C.); qfu@dicp.ac.cn (Q.F.)
}

Received: 7 May 2020; Accepted: 4 July 2020; Published: 14 July 2020

check for updates

\begin{abstract}
Vertically stacked hexagonal boron nitride (h-BN)/graphene heterostructures present potential applications in electronic, photonic, and mechanical devices, and their interface interaction is one of the critical factors that affect the performances. In this work, the vertical h-BN/graphene heterostructures with high coverage are synthesized by chemical vapor deposition (CVD) of h-BN on Ni substrates followed by segregation growth of graphene at the h-BN/Ni interfaces, which are monitored by in situ surface microscopy and surface spectroscopy. We find that h-BN overlayers can be decoupled from Ni substrates by the graphene interlayers. Furthermore, the h-BN domain boundaries exhibit a confinement effect on the graphene interlayer growth and the lower graphene domains are limited within the upper h-BN domains. This work provides new insights into the formation mechanism and interface interaction of the vertical heterostructures.
\end{abstract}

Keywords: hexagonal boron nitride (h-BN); graphene; vertical heterostructures; interface interaction

\section{Introduction}

Graphene shows promising applications in electronic, photonic and mechanical devices due to its excellent physical and chemical properties [1-4]. Usually, graphene overlayers need to be transferred onto $\mathrm{SiO}_{2}$ surfaces for device applications, while performances of the graphene-based devices are often limited by the $\mathrm{SiO}_{2}$ surfaces such as surface roughness and charging effect [5-7]. Hence, it is urgent to find suitable supports to replace $\mathrm{SiO}_{2}$. Hexagonal boron nitride (h-BN) has a similar structure to graphene, while it is an insulator with a bandgap of $5.8 \mathrm{eV}$, having desirable chemical inertness and mechanical strength $[8,9]$. h-BN can assemble with graphene to form h-BN/graphene heterostructures. Integration of h-BN and graphene in a vertically stacked way may achieve high-performance graphene-based devices, in which the atomically flat and dangling-bond-free $\mathrm{h}-\mathrm{BN}$ surface enables them to avoid the charge trapping at the interfaces [10,11].

Construction of well-defined h-BN/graphene heterostructures becomes critical for future potential applications. Chemical vapor deposition (CVD) is widely used to prepare hybrid systems. For example, growing $\mathrm{h}-\mathrm{BN}$ and graphene in sequence on $\mathrm{Cu}$ or $\mathrm{Ni}$ substrate has been attempted [12-16]. Some researchers have reported plasma-enhanced CVD to grow graphene overlayers directly on exfoliated h-BN [17]. In addition, the co-segregation method and temperature-triggered chemical conversion have been successfully applied to prepare the heterostructures $[18,19]$. The synthesis 
of h-BN/graphene heterostructures has been extensively explored, and interface interaction in the heterostructures is worth further investigation. In situ investigations become important to understand growth processes and interface structures.

In this study, vertically stacked h-BN/graphene heterostructures on Ni substrates were prepared by combining CVD with segregation methods, which were monitored by in situ X-ray photoemission electron microscopy (XPEEM) and local X-ray photoemission electron spectrum (local XPS). It has been demonstrated that the graphene interlayer decouples the interaction between the h-BN overlayer and the metal substrate, and the decoupling effect is enhanced with the increasing graphene layer number. Additionally, there is a confinement effect of $\mathrm{h}-\mathrm{BN}$ domain boundaries on the graphene growth underneath such that the monolayer graphene domains cannot extend beyond the h-BN boundaries.

\section{Experiments}

Most experiments were carried out in a photoemission electron microscopy and low-energy electron microscopy (PEEM/LEEM) system from Elmitec-GmbH (Clausthal-Zellerfeld, Germany), which includes a preparation chamber and a main chamber with the base pressure of $3 \times 10^{-10}$ Torr. In the LEEM mode, micro-region low electron energy diffraction ( $\mu$-LEED) can be performed to investigate surface structures from local regions. Low energy electron reflectivity (LEER) spectra, known as the intensity of elastically backscattered electron beam as a function of incident electron beam energy, can be applied to detect surface work function and the layer number of two-dimensional (2D) materials [20]. XPEEM measurements were conducted at the XPEEM endstation (BL09U, Dreamline) of Shanghai Synchrotron Radiation Facility (SSRF) in China. Energy-filtered XPEEM image stacks were collected to construct local XPS of small regions of interest (ROI) by measuring the integrated intensity of the ROI through the stack as a function of energy. The energy resolution of the local XPS is $0.2 \mathrm{eV}$, and binding energy positions were calibrated by the Fermi edge of each sample. A lateral resolution of XPEEM images down to about $20 \mathrm{~nm}$ can be achieved.

Ni foils (99.3\%, Goodfellow at Cambridge in England) were used as the substrates, which were cleaned by cycles of $\mathrm{Ar}^{+}$ion $\left(2.0 \mathrm{kV}, 7 \times 10^{-6}\right.$ Torr Ar) sputtering and annealing in ultrahigh vacuum (UHV) to $900^{\circ} \mathrm{C}$. The substrates contain a high concentration of carbon impurity at the near-surface region, acting as the carbon reservoir [21]. Carbon species segregated to the surface forming graphene overlayers when the surface temperature was decreased to $650^{\circ} \mathrm{C}$. If the temperature was increased to $700{ }^{\circ} \mathrm{C}$, the graphene overlayers were dissolved again. All h-BN overlayers were synthesized by exposure of the substrate surfaces to $1.0 \times 10^{-6}$ Torr borazine at $850^{\circ} \mathrm{C}$. When the temperature was decreased to 650 and $600{ }^{\circ} \mathrm{C}$ successively, graphene layers were segregated at h-BN/Ni interfaces, forming the vertically stacked h-BN/graphene heterostructures.

\section{Results}

Graphene overlayers were synthesized on the Ni substrates by surface segregation of near-surface carbon species as the temperature was decreased from 900 to $650{ }^{\circ} \mathrm{C}$ (Figure 1a). $\mu$-LEED patterns acquired from the forming graphene domains display a typical six-fold symmetric structure (Figure $1 \mathrm{~b}$ ) due to the 2D hexagonal lattice [22]. The LEER spectra (Figure 1c) show that graphene domains have one dip at the incident energy close to $2.0 \mathrm{eV}$, suggesting the monolayer feature [23,24]. All the results indicate that graphene can be prepared through the surface segregation of carbon species from near-surface regions of the Ni substrates. 

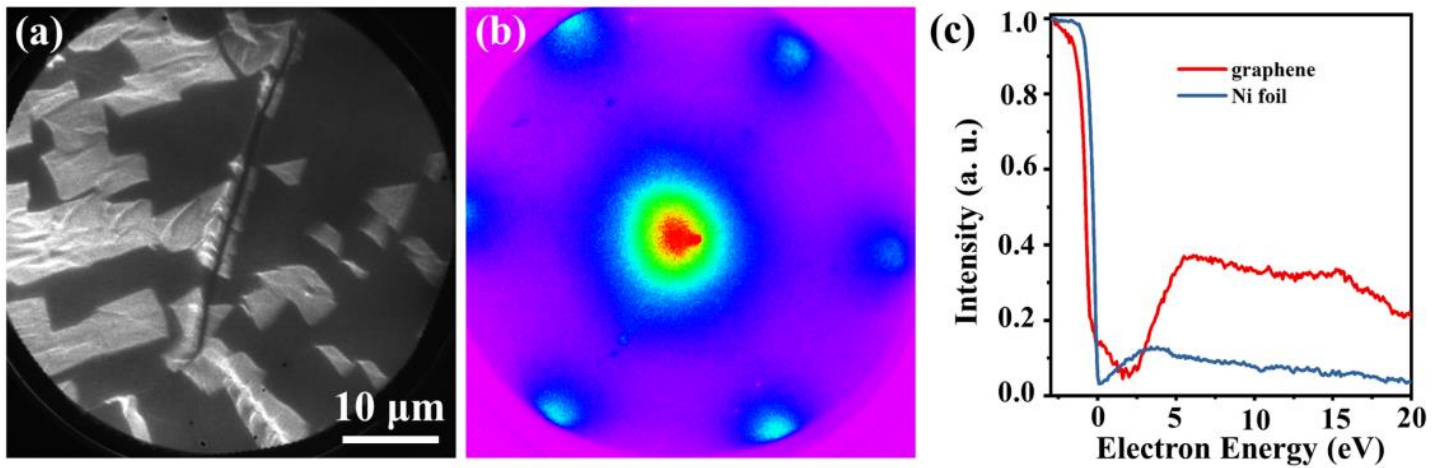

Figure 1. (a) LEEM image of graphene domains formed on the bared Ni surface at $650^{\circ} \mathrm{C}$. (b) $\mu$-LEED pattern $(50 \mathrm{eV})$ acquired from the forming graphene domains. (c) LEER spectra acquired from graphene domains and the bared Ni surface, respectively.

The growth of $\mathrm{h}-\mathrm{BN}$ overlayers was conducted by the CVD process $\left(1.0 \times 10^{-6}\right.$ Torr borazine, $850^{\circ} \mathrm{C}$ ). After keeping the growth time for $30 \mathrm{~min}$, a full h-BN layer was obtained (Figure 2a). $\mu$-LEED patterns (Figure 2b) of h-BN overlayers show a remarkable three-fold symmetric structure owing to its intrinsic symmetry $[25,26]$. LEER spectra indicate that h-BN overlayers have one dip at the incident energy of $2.0 \mathrm{eV}$, suggesting the monolayer feature $[27,28]$. Similarly, previous studies have confirmed that h-BN growth on Ni substrates is a monolayer self-limited process in which borazine molecules decompose to $\mathrm{B}$ and $\mathrm{N}$ containing intermediates and successively reassemble into h-BN overlayers [29].
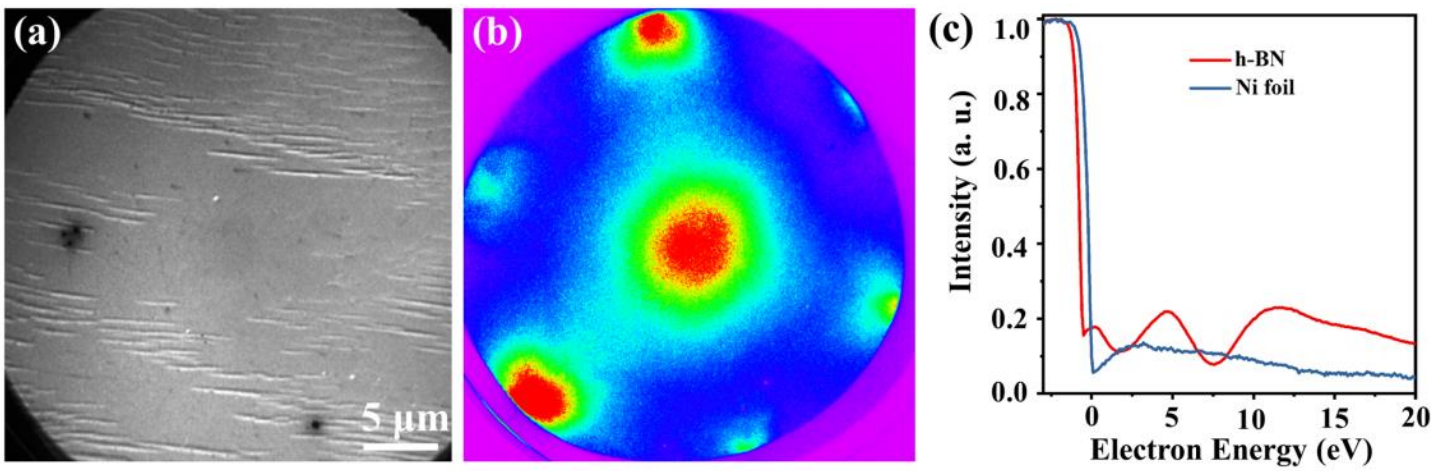

Figure 2. (a) LEEM image of full monolayer h-BN on the Ni substrate at $850{ }^{\circ} \mathrm{C}$. (b) $\mu$-LEED pattern $(50$ $\mathrm{eV}$ ) of the h-BN overlayer. (c) LEER spectra acquired from h-BN and Ni substrate, respectively.

From the surface covered by a full monolayer h-BN, graphene interlayers were segregated at the $\mathrm{h}-\mathrm{BN} / \mathrm{Ni}$ interface when decreasing the surface temperature, producing vertically stacked h-BN/graphene heterostructures. When the substrate temperature was cooled to $650{ }^{\circ} \mathrm{C}$ for several minutes, graphene domains with bright contrast appear at the h-BN/Ni interfaces, as displayed in the LEEM image (Figure 3a). The pure h-BN zone and the graphene segregation zone are labeled as zone 1 and zone 2 in Figure 3a, respectively. The two different zones can be easily distinguished by the PEEM image which is sensitive to the surface work function. The contrast of the graphene segregation zone (zone 2) is darker than the pure h-BN zone (zone 1), suggesting the higher work function of the graphene segregation zone (zone 2) (Figure 3e). Besides, LEER spectra results (Figure 3b) indicate that the difference in their work function is $0.5 \mathrm{eV}$, which agrees well with PEEM results. 

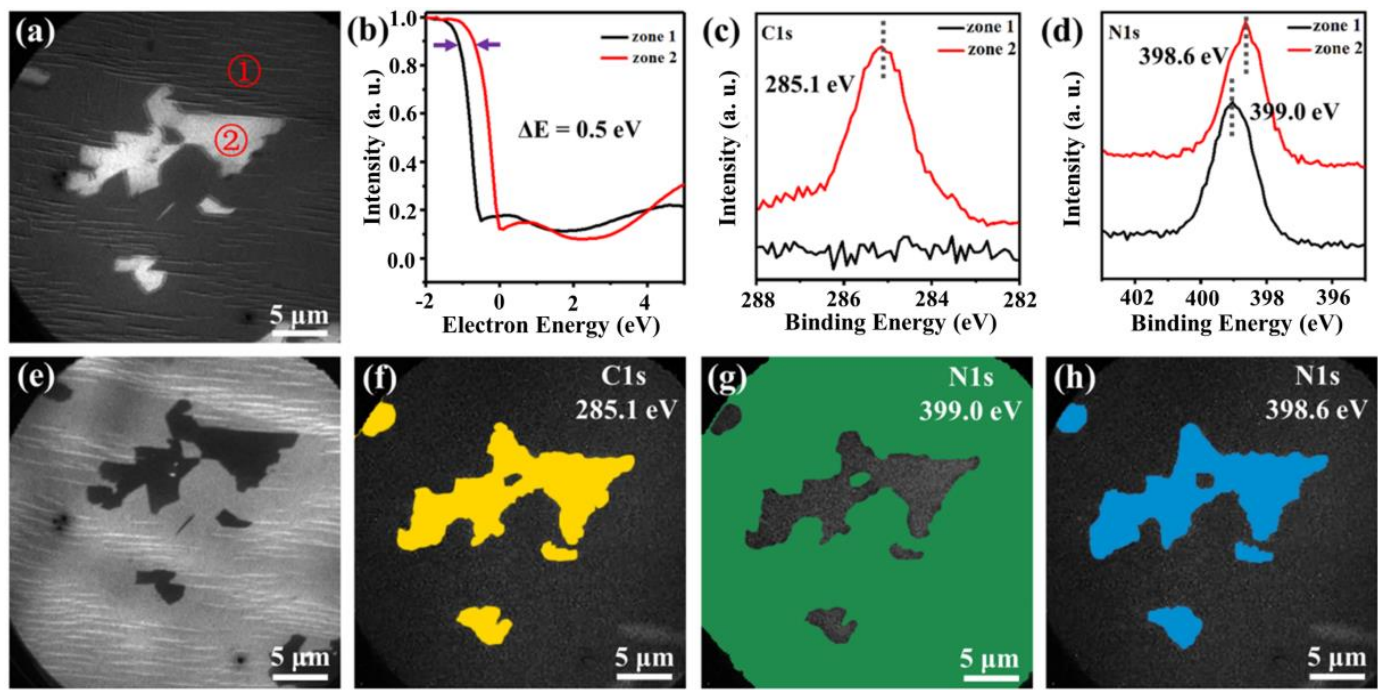

Figure 3. (a) LEEM and (e) PEEM images of the surfaces segregated at $650{ }^{\circ} \mathrm{C}$ (b) LEER spectra acquired from zone 1 and zone 2, respectively. (c) C 1 s and (d) N 1s core level spectra $(h v=500 \mathrm{eV})$ measured from zone 1 and zone 2, respectively. (f-h) XPEEM images at $C$ 1s core levels with binding energies of $285.1 \mathrm{eV}$, and N 1s core levels with binding energies of 399.0 and $398.6 \mathrm{eV}(h v=500 \mathrm{eV})$, respectively. The distribution of related elements with the certain chemical state is color-coded and the black contrast represents background signals, and those initial data are shown in Figure S1 in the supplementary materials.

Local XPS measurement was conducted to analyze the chemical state of the surface. Figure $3 c, d$ illustrates local C 1s and $\mathrm{N}$ 1s core level spectra acquired from the two zones. There are no $\mathrm{C} 1 \mathrm{~s}$ signals from the pure h-BN zone (zone 1), while a strong C 1s peak with binding energy at $285.1 \mathrm{eV}$ is seen from the graphene segregation zone (zone 2) which is from the single-layer epitaxial graphene [30]. $\mathrm{N} 1$ s binding energy of zone 1 is located at $399.0 \mathrm{eV}$, which adopts a typical monolayer feature of h-BN on $\mathrm{Ni}$ (Figure 2c) [15,31]. The $\mathrm{N}$ 1s binding energy of zone 2 is located at $398.6 \mathrm{eV}$, demonstrating $-0.4 \mathrm{eV}$ binding energy shift compared to that of zone 1 . These results confirm that h-BN in zone 2 interacts with the Ni substrates less strongly than zone 1 , suggesting that the $\mathrm{h}-\mathrm{BN} / \mathrm{Ni}$ interfaces are decoupled by the graphene interlayers. XPEEM images of $C$ 1s with binding energy of $285.1 \mathrm{eV}$ and $\mathrm{N}$ 1s with binding energy of 399.0 and $398.6 \mathrm{eV}$ are shown in Figure $2 \mathrm{f}-\mathrm{h}$, and the element distribution with the certain chemical state can be clearly observed in the field of view. Overall, we demonstrate the formation of vertical h-BN/monolayer graphene heterostructures on the Ni substrates.

For the device applications, it is necessary to grow large-area heterostructures. We find that decreasing the segregation temperature is an efficient way to increase the coverage of graphene interlayers, i.e., the vertically stacked h-BN/graphene heterostructures. Our previous studies have shown that nickel carbides form on Ni surfaces when the substrate temperature is lower than $450{ }^{\circ} \mathrm{C}$, but the carbide overlayers decompose and transform to graphene as the temperature is higher than $500{ }^{\circ} \mathrm{C}$ [30]. Accordingly, in order to drive more carbon species to segregate on the surface, the successive segregation temperature of $600^{\circ} \mathrm{C}$ was chosen. When keeping this temperature for $30 \mathrm{~min}$, graphene overlayers almost approach a full coverage (Figure $4 \mathrm{a}$ ). In such a case, graphene segregation zones display two different contrasts in the LEEM image, which are labeled by zone 4 and zone 5, respectively. The remaining pure h-BN zones are marked by zone 3. LEER spectra results (Figure $4 \mathrm{~b}$ ) indicate that the work function of zone 5 is $0.4 \mathrm{eV}$ higher than zone 4 and that of zone 4 is $0.5 \mathrm{eV}$ higher than zone 3. 

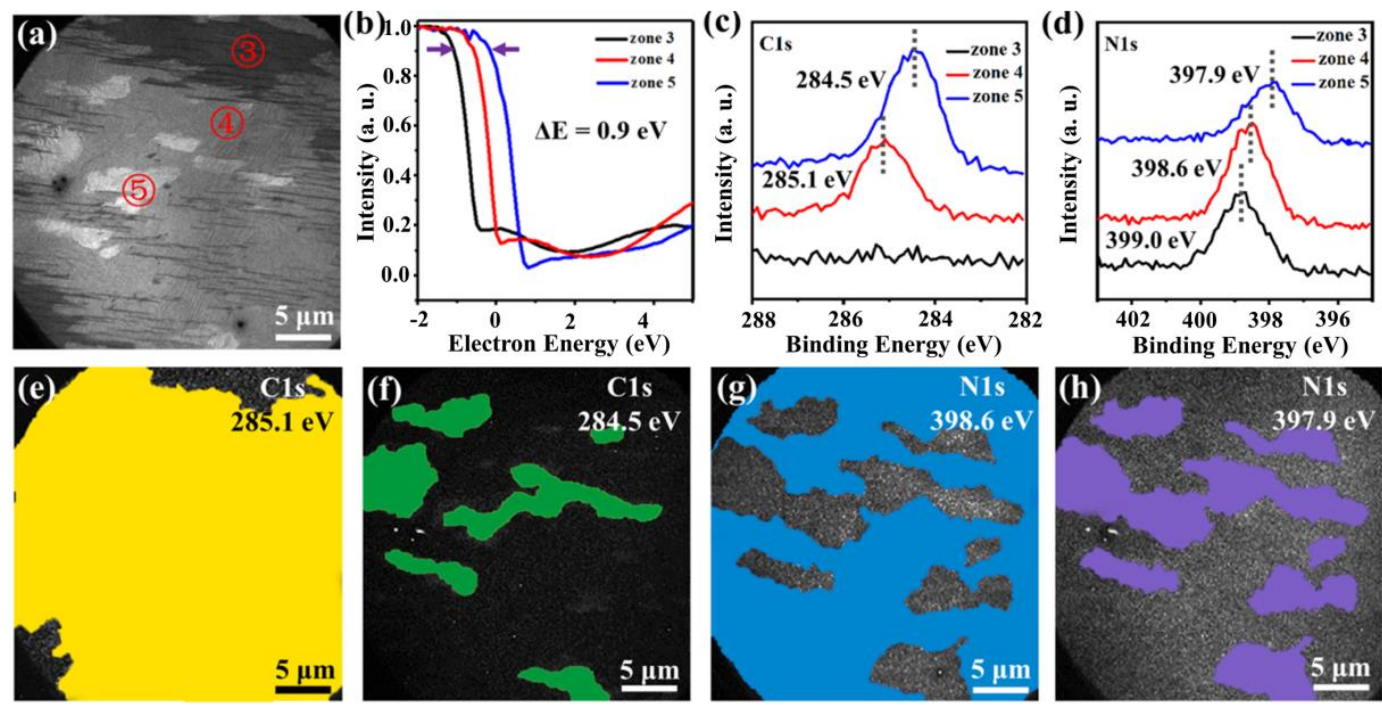

Figure 4. (a) LEEM image of the surfaces successively segregated at $600^{\circ} \mathrm{C}$. (b) LEER spectra acquired from zone 3, 4, and 5, respectively. (c) C 1s and (d) N 1s core level spectra ( $h v=500 \mathrm{eV}$ ) measured on zone 3, 4 and 5, respectively. (e-h) XPEEM images at $C 1$ s core levels with binding energies of 285.1 and $284.5 \mathrm{eV}$, and N 1s core levels with binding energies of 398.6 and $397.9 \mathrm{eV}$, respectively. The distribution of related elements with the certain chemical state is color-coded and the black contrast represents background signals, and those initial data are shown in Figure S2 in the supplementary materials.

Local XPS and XPEEM characterization were applied to analyze the three representative zones. $\mathrm{N}$ 1s binding energy in zone 3 is located at $399.0 \mathrm{eV}$ (Figure 4d) in accordance with the pure h-BN structure. In zone 4, binding energies of $\mathrm{N}$ 1s and $\mathrm{C}$ 1s peaks are $398.6 \mathrm{eV}$ (Figure 4d) and $285.1 \mathrm{eV}$ (Figure 4c), respectively, which are corresponding to those of h-BN/monolayer-graphene heterostructures abovementioned. In zone 5, C 1s spectrum is peaked at $284.5 \mathrm{eV}$ (Figure 4c), shifting down by $0.6 \mathrm{eV}$ compared with that in zone 4 , which is close to that of highly oriented pyrolytic graphite (HOPG) [32]. Therefore, graphene overlayers in zone 5 can be ascribed to multilayer structure, which further combine with the upper h-BN layer to form h-BN/multilayer graphene heterostructures [32,33]. The binding energy peak of $\mathrm{N} 1 \mathrm{~s}$ at $397.9 \mathrm{eV}$ in zone 5 (Figure $4 \mathrm{~d}$ ) shows a BE shift of $-0.7 \mathrm{eV}$ compared with $\mathrm{N} 1 \mathrm{~s}$ in the h-BN/monolayer-graphene heterostructure zone, implying that the h-BN/Ni interface interaction in zone 5 is weaker than that in zone 4-that is to say, multilayer graphene overlayers enhance the decoupling effect on h-BN/Ni interfaces in comparison with monolayer graphene. In addition, XPEEM images of $\mathrm{C} 1 \mathrm{~s}$ with binding energy of 285.1 and $284.5 \mathrm{eV}$, and $\mathrm{N} 1 \mathrm{~s}$ with binding energy of 398.6 and $397.9 \mathrm{eV}$ are shown in Figure $4 \mathrm{e}-\mathrm{h}$, and the element distribution with the certain chemical state can be clearly displayed in the field of view.

Similar graphene segregation was performed on the Ni surface covered by sub-monolayer h-BN. First, h-BN domains were grown by exposure to $1.0 \times 10^{-6}$ Torr borazine at $850{ }^{\circ} \mathrm{C}$. The domains display a triangle shape or blade shape due to the intrinsic three-fold symmetry structure, as shown in Figure 5a [25]. When the temperature was decreased to $680{ }^{\circ} \mathrm{C}$, dark contrast domains appear at the h-BN domains (Figure $5 \mathrm{~b}$ ), indicating the formation of the segregated graphene domains. Upon decreasing the temperature to $600^{\circ} \mathrm{C}$, higher coverage graphene domains form (Figure 5c).

Graphene domains form at three different sites, namely, the h-BN/Ni interfaces (labeled as zone B in Figure 5d), the h-BN domain boundaries (labeled as zone $C$ in Figure $5 d$ ) and the bared Ni surfaces (labeled as zone D in Figure 5d). Hence, vertically stacked h-BN/graphene heterostructures and in-plane h-BN/graphene heterostructures are simultaneously synthesized on the surfaces, respectively. Especially for the vertical h-BN/graphene heterostructures, the graphene domains under h-BN cannot extend out of each upper h-BN domain, even after a segregation time of $153 \mathrm{~min}$, as shown by Figure $5 \mathrm{c}, \mathrm{d}$. While on the bared Ni surfaces, graphene domains grow without impedance like the zone $\mathrm{B}$. The results suggest that graphene domains underneath $\mathrm{h}-\mathrm{BN}$ are trapped by $\mathrm{h}-\mathrm{BN}$ domain boundaries. 

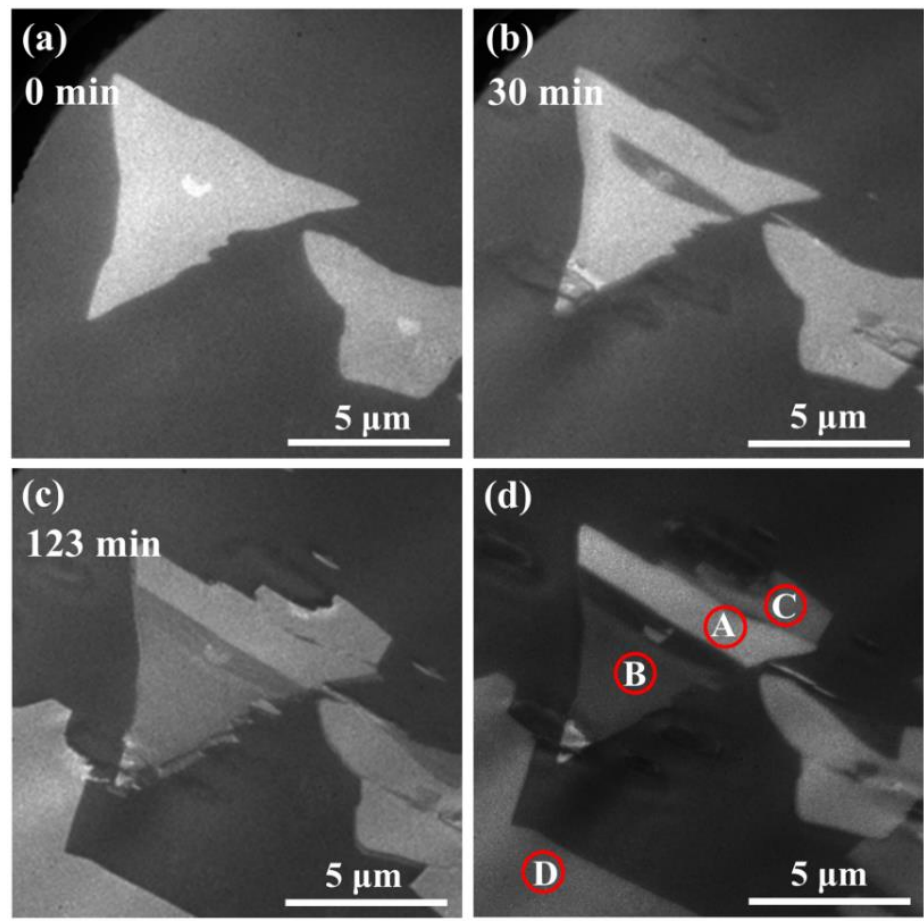

Figure 5. (a-c) LEEM images of graphene segregation processes on the surface covered by sub-monolayer h-BN. (d) UV-PEEM image of the surfaces. The labeled zones A, B, C and D represent pure h-BN, graphene segregated under h-BN, at the h-BN domain boundaries and on bared Ni zones, respectively.

\section{Discussion}

Carbon solubility at the near-surface region of $\mathrm{Ni}$ substrates plays an important role in the formation of the vertically stacked h-BN/graphene heterostructures. On the other hand, the segregation temperature is crucial to the heterostructure formation as well. Carbon atoms cannot migrate to the surface and form graphene with temperatures higher than $700{ }^{\circ} \mathrm{C}$. When the temperature is lower than $450{ }^{\circ} \mathrm{C}$, carbon atoms react with nickel to form the surface nickel carbide phase [30,34]. Hence, temperatures between 600 and $700{ }^{\circ} \mathrm{C}$ are the most appropriate for the graphene segregation. Similar to Ni substrates, other metal substrates which can dissolve a certain amount of $C$ such as $C o$ and Ru can be used to achieve the vertical h-BN/graphene heterostructures by the method combining CVD and segregation under the appropriate temperature [35]. We also reveal how the interlayer decouples the interface interaction between metal substrates and 2D materials. As shown in Scheme 1a, the interface between $\mathrm{h}-\mathrm{BN}$ and the Ni substrate is strongly decoupled with the increase in graphene layer number. Therefore, the control of the segregated graphene layers is the key step to modulate the decoupling at the h-BN/Ni interface.

Additionally, on the surface covered by sub-monolayer h-BN, segregated graphene layers are confined within each h-BN domain. The h-BN domain acts like a clap over graphene, restricting the graphene domain as shown in Scheme 1b. Previous reports have proved that boundaries of h-BN or graphene domains are coordinatively unsaturated and thus bind more strongly with the metal substrate surface such that boundaries tightly attach to the surface and twist downward [36,37]. These twisting domain boundaries combine with the substrate surfaces to form a confinement space. Accordingly, the h-BN domain boundary serves as a barrier to impede the growth of the underneath graphene, showing a boundary confinement effect. If the concentration of near-surface $C$ species is high enough, it is possible to form the multilayer graphene, which enlarges the h-BN/Ni interface distance. The interaction between the h-BN domain boundary and Ni surfaces can be weakened such that forming graphene multilayers may extend outside the h-BN domains. Therefore, the domain boundary confinement effect is expected to be weakened as the concentration of near-surface $\mathrm{C}$ species increases. 
(a)

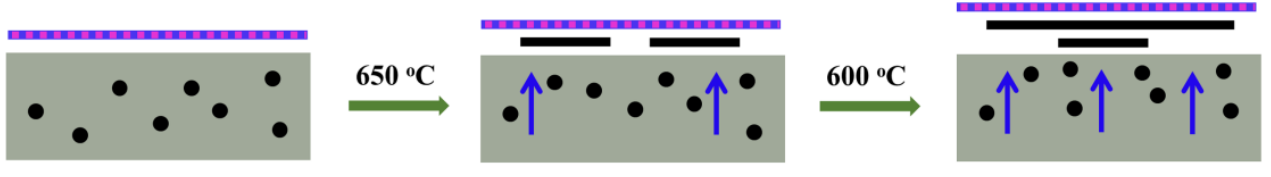

(b)

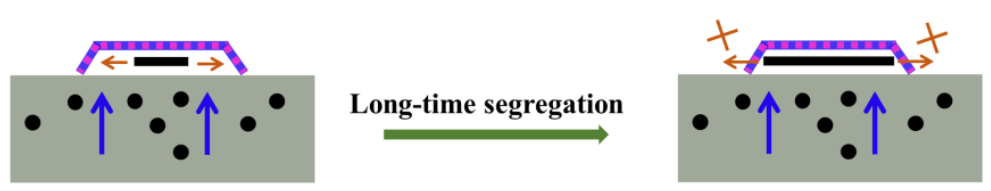

Scheme 1. (a) The schematic diagrams of vertically stacked h-BN/graphene heterostructure growth processes and the decoupling effect of graphene interlayers on $\mathrm{h}-\mathrm{BN} / \mathrm{Ni}$ interfaces in the case of the surfaces covered by full monolayer h-BN. (b) The schematic diagrams of the confined effect of h-BN domain boundaries on the underneath segregated graphene in the case of the surfaces covered by sub-monolayer h-BN.

\section{Conclusions}

The vertically stacked $\mathrm{h}$-BN/graphene heterostructures are prepared by exposure of the substrates to $1.0 \times 10^{-6}$ Torr borazine at $850{ }^{\circ} \mathrm{C}$ (CVD method) to grow h-BN on Ni substrates followed by segregation growth of graphene at $\mathrm{h}-\mathrm{BN} / \mathrm{Ni}$ interfaces around $600{ }^{\circ} \mathrm{C}$. The segregation temperature acts as the key factor to control the integration of the heterostructures. The whole growth processes are monitored by in situ LEEM and surface spectroscopy. The synchrotron-based local XPS and XPEEM results reveal that the stacking order of the heterostructure is $\mathrm{h}$-BN upper on graphene and the monolayer and multilayer graphene structures both form underneath the h-BN. The h-BN/Ni interfaces are decoupled by the graphene interlayers, and the decoupling effect is enhanced by the thicker graphene layers. Furthermore, it has been revealed that the segregated graphene layers underneath h-BN hardly grow across h-BN domain boundaries due to the strong boundary-substrate interaction, indicating a typical boundary confinement effect. Our work provides a more detailed understanding of the fabrication, the stacked structure and the interface interaction for h-BN/graphene heterostructures, which can be expected to facilitate synthesis and applications of van der Waals heterostructures.

Supplementary Materials: The followings are available online at http:/www.mdpi.com/2571-9637/3/3/24/s1. Figure S1: Initial XPEEM images at C 1s and N 1s core levels with binding energies of 285.1, 399.0, and $398.6 \mathrm{eV}$ $(h v=500 \mathrm{eV})$ at the surface segregation temperature of $650^{\circ} \mathrm{C}$. Figure S2: Initial XPEEM images at $\mathrm{C} 1 \mathrm{~s}$ core levels with binding energies of 285.1 and $284.5 \mathrm{eV}$, and $\mathrm{N} 1$ s core levels with binding energies of 398.6 and $397.9 \mathrm{eV}$ at the surface segregation temperature of $600{ }^{\circ} \mathrm{C}$.

Author Contributions: Conceptualization, W.W., Q.F. and Y.C.; formal analysis, Q.F., W.W., Y.C. and G.Z.; investigation, W.W. and G.Z.; writing —original draft preparation, W.W. and J.P.; writing-review and editing, Q.F., Y.C. and W.W.; resources, Q.F. and G.Z.; funding, Q.F., Y.C. and W.W. All authors have read and agreed to the published version of the manuscript.

Funding: This work was financially supported by the National Natural Science Foundation of China (No. 21688102, No. 21825203 and No. 21872169), the National Key R\&D Program of China (No. 2016YFA0200200 and No. 2017YFB0602205), Strategic Priority Research Program of the Chinese Academy of Sciences (Grant No. XDB17020000), Natural Science Foundation of Jiangsu Province (No. BK20170426) and China Postdoctoral Science Foundation (No. 2019M651997).

Conflicts of Interest: The authors declare no conflict of interest.

\section{References}

1. Schwierz, F. Graphene transistors. Nat. Nanotechnol. 2010, 5, 487-496. [CrossRef] [PubMed]

2. Chen, J.-H.; Ishigami, M.; Jang, C.; Hines, D.R.; Fuhrer, M.S.; Williams, E.D. Printed graphene circuits. Adv. Mater. 2007, 19, 3623. [CrossRef] 
3. Bonaccorso, F.; Sun, Z.; Hasan, T.; Ferrari, A.C. Graphene photonics and optoelectronics. Nat. Photonics 2010, 4, 611-622. [CrossRef]

4. Stoeberl, U.; Wurstbauer, U.; Wegscheider, W.; Weiss, D.; Eroms, J. Morphology and flexibility of graphene and few-layer graphene on various substrates. Appl. Phys. Lett. 2008, 93, 051906. [CrossRef]

5. Ishigami, M.; Chen, J.H.; Cullen, W.G.; Fuhrer, M.S.; Williams, E.D. Atomic structure of graphene on $\mathrm{SiO}_{2}$. Nano Lett. 2007, 7, 1643-1648. [CrossRef] [PubMed]

6. Chen, J.-H.; Jang, C.; Xiao, S.; Ishigami, M.; Fuhrer, M.S. Intrinsic and extrinsic performance limits of graphene devices on $\mathrm{SiO}_{2}$. Nat. Nanotechnol. 2008, 3, 206-209. [CrossRef] [PubMed]

7. Ando, T. Screening effect and impurity scattering in monolayer graphene. J. Phys. Soc. Jpn. 2006, 75, 074716. [CrossRef]

8. Kho, J.-G.; Moon, K.-T.; Kim, J.-H.; Kim, D.-P. Properties of boron nitride films produced by the spin-coating process of polyborazine. J. Am. Ceram. Soc. 2000, 83, 2681-2683. [CrossRef]

9. Kim, K.K.; Hsu, A.; Jia, X.; Kim, S.M.; Shi, Y.; Hofmann, M.; Nezich, D.; Rodriguez-Nieva, J.F.; Dresselhaus, M.; Palacios, T.; et al. Synthesis of monolayer hexagonal boron nitride on $\mathrm{Cu}$ foil using chemical vapor deposition. Nano Lett. 2012, 12, 161-166. [CrossRef]

10. Xue, J.; Sanchez-Yamagishi, J.; Bulmash, D.; Jacquod, P.; Deshpande, A.; Watanabe, K.; Taniguchi, T.; Jarillo-Herrero, P.; Leroy, B.J. Scanning tunnelling microscopy and spectroscopy of ultra-flat graphene on hexagonal boron nitride. Nat. Mater. 2011, 10, 282-285. [CrossRef]

11. Ling, X.; Fang, W.; Lee, Y.-H.; Araujo, P.T.; Zhang, X.; Rodriguez-Nieva, J.F.; Lin, Y.; Zhang, J.; Kong, J.; Dresselhaus, M.S. Raman enhancement effect on two-dimensional layered materials: Graphene, h-BN and $\mathrm{MoS}_{2}$. Nano Lett. 2014, 14, 3033-3040. [CrossRef] [PubMed]

12. Liu, Z.; Song, L.; Zhao, S.; Huang, J.; Ma, L.; Zhang, J.; Lou, J.; Ajayan, P.M. Direct growth of graphene/hexagonal boron nitride stacked layers. Nano Lett. 2011, 11, 2032-2037. [CrossRef] [PubMed]

13. Kim, S.M.; Hsu, A.; Araujo, P.T.; Lee, Y.-H.; Palacios, T.; Dresselhaus, M.; Idrobo, J.-C.; Kim, K.K.; Kong, J. Synthesis of patched or stacked graphene and h-BN flakes: A route to hybrid structure discovery. Nano Lett. 2013, 13, 933-941. [CrossRef]

14. Song, X.; Gao, T.; Nie, Y.; Zhuang, J.; Sun, J.; Ma, D.; Shi, J.; Lin, Y.; Ding, F.; Zhang, Y.; et al. Seed-assisted growth of single-crystalline patterned graphene domains on hexagonal boron nitride by chemical vapor deposition. Nano Lett. 2016, 16, 6109-6116. [CrossRef] [PubMed]

15. Yang, Y.; Fu, Q.; Li, H.; Wei, M.; Xiao, J.; Wei, W.; Bao, X. Creating a nanospace under an h-BN cover for adlayer growth on nickel(111). ACS Nano 2015, 9, 11589-11598. [CrossRef]

16. Li, Q.; Zhao, Z.; Yan, B.; Song, X.; Zhang, Z.; Li, J.; Wu, X.; Bian, Z.; Zou, X.; Zhang, Y.; et al. Nickelocene-precursor-facilitated fast growth of graphene/h-BN vertical heterostructures and its applications in OLEDs. Adv. Mater. 2017, 29, 1701325. [CrossRef] [PubMed]

17. Yang, W.; Chen, G.; Shi, Z.; Liu, C.-C.; Zhang, L.; Xie, G.; Cheng, M.; Wang, D.; Yang, R.; Shi, D.; et al. Epitaxial growth of single-domain graphene on hexagonal boron nitride. Nat. Mater. 2013, 12, 792. [CrossRef]

18. Zhang, C.; Zhao, S.; Jin, C.; Koh, A.L.; Zhou, Y.; Xu, W.; Li, Q.; Xiong, Q.; Peng, H.; Liu, Z. Direct growth of large-area graphene and boron nitride heterostructures by a co-segregation method. Nat. Commun. 2015, 6, 6519. [CrossRef]

19. Gao, T.; Song, X.; Du, H.; Nie, Y.; Chen, Y.; Ji, Q.; Sun, J.; Yang, Y.; Zhang, Y.; Liu, Z. Temperature-triggered chemical switching growth of in-plane and vertically stacked graphene-boron nitride heterostructures. Nat. Commun. 2015, 6, 6835. [CrossRef]

20. Murata, Y.; Starodub, E.; Kappes, B.B.; Ciobanu, C.V.; Bartelt, N.C.; McCarty, K.F.; Kodambaka, S. Orientation-dependent work function of graphene on Pd(111). Appl. Phys. Lett. 2010, 97, 143114. [CrossRef]

21. Batzill, M. The surface science of graphene: Metal interfaces, CVD synthesis, nanoribbons, chemical modifications, and defects. Surf. Sci. Rep. 2012, 67, 83-115. [CrossRef]

22. Wu, B.; Geng, D.; Xu, Z.; Guo, Y.; Huang, L.; Xue, Y.; Chen, J.; Yu, G.; Liu, Y. Self-organized graphene crystal patterns. NPG Asia Mater. 2013, 5, e36. [CrossRef]

23. Sutter, P.; Sadowski, J.T.; Sutter, E. Graphene on Pt(111): Growth and substrate interaction. Phys. Rev. B 2009, 80, 245411. [CrossRef] 
24. Hibino, H.; Wang, S.; Orofeo, C.M.; Kageshima, H. Growth and low-energy electron microscopy characterizations of graphene and hexagonal boron nitride. Prog. Cryst. Growth Charact. Mater. 2016, 62, 155-176. [CrossRef]

25. Yang, Y.; Fu, Q.; Wei, M.; Bluhm, H.; Bao, X. Stability of BN/metal interfaces in gaseous atmosphere. Nano Res. 2015, 8, 227-237. [CrossRef]

26. Liu, Y.; Bhowmick, S.; Yakobson, B.I. BN white graphene with "colorful" edges: The energies and morphology. Nano Lett. 2011, 11, 3113-3116. [CrossRef]

27. Mende, P.C.; Gao, Q.; Ismach, A.; Chou, H.; Widom, M.; Ruoff, R.; Colombo, L.; Feenstra, R.M. Characterization of hexagonal boron nitride layers on nickel surfaces by low-energy electron microscopy. Surf. Sci. 2017, 659, 31-42. [CrossRef]

28. Wei, W.; Lin, L.; Zhang, G.; Ye, X.; Bin, R.; Meng, C.; Ning, Y.; Fu, Q.; Bao, X. Effect of near-surface dopants on the epitaxial growth of h-BN on metal surfaces. Adv. Mater. Interfaces 2019, 6, 1801906. [CrossRef]

29. Auwarter, W.; Muntwiler, M.; Osterwalder, J.; Greber, T. Defect lines and two-domain structure of hexagonal boron nitride films on Ni(111). Surf. Sci. 2003, 545, L735-L740. [CrossRef]

30. Wei, M.; Fu, Q.; Yang, Y.; Wei, W.; Crumlin, E.; Bluhm, H.; Bao, X. Modulation of surface chemistry of CO on $\mathrm{Ni}(111)$ by surface graphene and carbidic carbon. J. Phys. Chem. C 2015, 119, 13590-13597. [CrossRef]

31. Preobrajenski, A.B.; Vinogradov, A.S.; Martensson, N. Monolayer of h-BN chemisorbed on Cu(111) and $\mathrm{Ni}(111)$ : The role of the transition metal 3d states. Surf. Sci. 2005, 582, 21-30. [CrossRef]

32. Blyth, R.I.R.; Buqa, H.; Netzer, F.P.; Ramsey, M.G.; Besenhard, J.O.; Golob, P.; Winter, M. XPS studies of graphite electrode materials for lithium ion batteries. Appl. Surf. Sci. 2000, 167, 99-106. [CrossRef]

33. Weatherup, R.S.; Amara, H.; Blume, R.; Dlubak, B.; Bayer, B.C.; Diarra, M.; Bahri, M.; Cabrero-Vilatela, A.; Caneva, S.; Kidambi, P.R.; et al. Interdependency of subsurface carbon distribution and graphene-catalyst interaction. J. Am. Chem. Soc. 2014, 136, 13698-13708. [CrossRef]

34. Addou, R.; Dahal, A.; Sutter, P.; Batzill, M. Monolayer graphene growth on Ni(111) by low temperature chemical vapor deposition. Appl. Phys. Lett. 2012, 100, 021601. [CrossRef]

35. Seah, C.-M.; Chai, S.-P.; Mohamed, A.R. Mechanisms of graphene by chemical vapour deposition on transition metals. Carbon 2014, 70,1-21. [CrossRef]

36. Lu, J.; Yeo, P.S.E.; Zheng, Y.; Xu, H.; Gan, C.K.; Sullivan, M.B.; Castro Neto, A.H.; Loh, K.P. Step flow versus mosaic film growth in hexagonal boron nitride. J. Am. Chem. Soc. 2013, 135, 2368-2373. [CrossRef] [PubMed]

37. Vlassiouk, I.; Regmi, M.; Fulvio, P.F.; Dai, S.; Datskos, P.; Eres, G.; Smirnov, S. Role of hydrogen in chemical vapor deposition growth of large single-crystal graphene. ACS Nano 2011, 5, 6069-6076. [CrossRef]

(C) 2020 by the authors. Licensee MDPI, Basel, Switzerland. This article is an open access article distributed under the terms and conditions of the Creative Commons Attribution (CC BY) license (http://creativecommons.org/licenses/by/4.0/). 